\title{
Temperatura base para emissão de folhas e nós, filocrono e plastocrono das plantas daninhas papuã e corriola
}

\author{
Base temperature for leaf and node appearance, phyllochron and plastochron in the weeds \\ Alexander grass and Morning glory
}

\author{
Gizelli Moiano de Paula' ${ }^{I}$ Nereu Augusto Streck ${ }^{\text {II }}$
}

\section{RESUMO}

O papuã (Brachiaria plantaginea) e a corriola (Ipomoea triloba) são importantes plantas daninhas de culturas anuais. O filocrono (intervalo de tempo entre o aparecimento de duas folhas sucessivas) e o plastocrono (intervalo de tempo entre o aparecimento de dois nós sucessivos) são importantes parâmetros do desenvolvimento vegetal. A soma térmica é usada para expressar tempo no filocrono e no plastocrono e o seu cálculo implica o conhecimento da temperatura base. $O$ objetivo do trabalho foi estimar a temperatura base para emissão de folhas e nós, o filocrono e o plastocrono em papuã e corriola, respectivamente. Os dados usados no trabalho são provenientes de um experimento com milho realizado em Santa Maria, RS, em várias épocas durante o ano agrícola 2005/ 2006. Em cada parcela de milho, foram deixadas crescer duas plantas de papuã e duas plantas de corriola. Na haste principal destas plantas, foram medidos, uma vez por semana, o Estágio de Haun (HS) no papuã e o número de nós visíveis (NN) na corriola. A temperatura base estimada usando a metodologia do menor valor de Quadrado Médio do Erro (QME) foi de $3^{\circ} \mathrm{C}$ para o papuã e $7^{\circ} \mathrm{C}$ para corriola. O filocrono em papuã variou de $100,1^{\circ} \mathrm{C}$ dia folha ${ }^{-1}$ a $142,6^{\circ} \mathrm{C}$ dia folha ${ }^{-1}$ em função da época do cultivo do milho e o plastocrono em corriola não diferiu entre épocas de cultivo sendo, em média, $38,8^{\circ} \mathrm{C}$ dia $n o^{-1}$.

Palavras-chave: desenvolvimento vegetal, soma térmica, Brachiaria plantaginea, Ipomoea triloba.

\section{ABSTRACT}

Alexander grass (Brachiaria plantaginea) and Morning glory (Ipomoea triloba) are important weeds in annual crops. The phyllochron (time interval between the appearance of two successive leaves) and the plastochron (time interval between the appearance of successive nodes) are important parameters of plant development. Thermal time is used to express time in the phyllochron and in the plastochron, and in the calculation of thermal time the value of the base temperature is needed. The objective of this study was to estimate the base temperature for leaf and node appearance, the phyllochron and the plastochron for Alexander grass and Morning glory, respectively. Data used in this paper come from an experiment with maize sown in several dates, carried out in Santa Maria, RS, Brazil, during the 2005/2006 growing season. Two plants of Alexander grass and two plants of Morning glory were left to grow in each maize plot. The main stem Haun Stage (HS) in Alexander grass and the number of visible nodes in Morning glory were measured on a weekly basis. Using the Mean Square Error (MSE) approach, base temperature was estimated as $3^{\circ} \mathrm{C}$ for Alexander and $7^{\circ} \mathrm{C}$ for Morning glory. The phyllochron in Alexander grass varied from $100.1^{\circ} \mathrm{C}$ day leaf ${ }^{1}$ to $142.6^{\circ} \mathrm{C}$ day leaf ${ }^{1}$ as a function of maize sowing date. The plastochron in Morning glory did not vary among maize sowing dates, with an average value of $38.8^{\circ} \mathrm{C}$ day node $e^{-1}$.

Key words: plant development, thermal time, Brachiaria plantaginea, Ipomoea triloba.

\section{INTRODUÇÃO}

O papuã (Brachiaria plantaginea (Link) Hitchc), família Poaceae, é uma planta anual com reprodução por sementes. Seu ciclo de desenvolvimento ocorre principalmente durante a primavera e o verão como planta daninha em áreas com culturas anuais, especialmente lavouras de milho ou soja (KISSMANN \& GROTH, 1999). A espécie tem sido apontada como uma das poaceas mais importante no Brasil, pois é uma

IPrograma de Pós-graduação em Engenharia Agrícola, Universidade Federal de Santa Maria (UFSM), Santa Maria, RS, Brasil.

"Departamento de Fitotecnia, Centro de Ciências Rurais (CCR), UFSM. Av. Roraima, 1000, Campus Universitário, Camobi, 97105-900, Santa Maria, RS, Brasil. E-mail: nstreck2@yahoo.com.br. Autor para correspondência. 
competidora por espaço, luz, água e minerais com culturas anuais como a soja, resultando em perdas no rendimento dessa cultura (PIRES et al., 2001; FLECK et al., 2002). A corriola (Ipomoea triloba L.), família Convolvulaceae, é uma planta anual nativa da América do Sul, que se reproduz por sementes. I. triloba é uma das espécies de Ipomoea mais comum como planta daninha em lavouras de culturas anuais em quase todas as regiões do Brasil (KISSMANN \& GROTH, 1999).

A caracterização do desenvolvimento das plantas daninhas é um importante passo na elaboração e na definição de estratégias de manejo visando seu controle. O desenvolvimento vegetal refere-se a eventos que envolvem desde diferenciação celular, iniciação (organogênese), aparecimento de órgãos (morfogênese) e estende-se até a senescência da planta (HODGES, 1991). O desenvolvimento depende de vários fatores ambientais, sendo que a temperatura do ar é o principal elemento meteorológico que afeta o desenvolvimento dos vegetais (STRECK, 2002; GRAMIG \& STOLTENBERG, 2007). Para relacionar desenvolvimento com temperatura do ar, é muito utilizado o conceito da soma térmica ou dos graus-dia. Na clássica equação de cálculo da soma térmica, são acumulados os valores de temperatura média diária do ar acima de uma temperatura base (Tb), considerada a temperatura abaixo da qual não ocorre desenvolvimento ou este é tão lento que, para fins de simulação do desenvolvimento vegetal, pode ser considerado desprezível (McMASTER \& WILHELM, 1997).

A grande diversidade de espécies daninhas faz com que ainda não se conheça a Tb de algumas delas (GRAMIG \& STOLTENBERG, 2007). Em algumas regiões do Brasil, o papuã é usado como uma planta forrageira, assim como outras Brachiarias, tais como a Brachiaria brizantha e a Brachiaria decumbens. A $\mathrm{Tb}$ dessas duas últimas espécies são $15^{\circ} \mathrm{C}$ e $17^{\circ} \mathrm{C}$, respectivamente (MENDONÇA \& RASSINI, 2006). Para a Tb em papuã e corriola, o único registro encontrado na literatura foi $10^{\circ} \mathrm{C}$ para taxa de aparecimento de folhas (STRECK, 2006). Esse valor, no entanto, foi assumido pelo autor, e não estimado por similaridade com a Tb de cultivos de verão como milho e soja.

A velocidade de emissão de folhas ou de nós, em uma haste ou na planta toda, é um importante parâmetro do desenvolvimento vegetal. Ao se integrar a velocidade de emissão de folhas ou nós no tempo, têm-se o número de folhas (NF) ou de nós (NN) acumulados, respectivamente, os quais são uma excelente medida do desenvolvimento vegetal (XUE et al., 2004). O NF e o NN estão também associados à evolução da área foliar da planta, a qual intercepta radiação solar usada na fotossíntese para produção de fitomassa e no processo de evapotranspiração da cultura (STRECK, 2002; XUE et al., 2004).

Em modelos matemáticos, uma das maneiras bastante utilizadas para calcular NF é por meio do conceito de filocrono, definido como o intervalo de tempo entre o aparecimento de duas folhas sucessivas na haste principal, tendo como unidade ${ }^{\circ} \mathrm{C}$ dia folha ${ }^{-1}$ (McMASTER et al., 1991; STRECK et al., 2004, 2005a; GRAMIG \& STOLTENBERG, 2007). O filocrono é mais usado em gramíneas que em dicotiledôneas, sendo que o NN é o parâmetro de desenvolvimento vegetativo mais usado nas dicotiledôneas (SINCLAIR, 1984; BAKER \& REDDY, 2001). Para calcular o NN, usa-se o conceito de plastocrono, que é o intervalo de tempo entre o aparecimento de dois nós sucessivos em uma haste, com unidade ${ }^{\circ} \mathrm{C}$ dia nó ${ }^{-1}$ (BAKER \& REDDY, 2001; STRECK et al., 2002; STRECK et al., 2005b).

Frente ao exposto, é importante estimar a $\mathrm{Tb}$ dessas duas invasoras e determinar o filocrono e o plastocrono a fim de se ter maior precisão na simulação do desenvolvimento destas duas espécies de plantas daninhas. O objetivo do trabalho foi estimar a temperatura base para emissão de folhas e nós, o filocrono e o plastocrono em papuã e corriola, respectivamente.

\section{MATERIAL E MÉTODOS}

Os dados usados neste trabalho são provenientes de um experimento de várias épocas de semeadura de milho (variedade BRS Missões), conduzido na área experimental do Departamento de Fitotecnia, Universidade Federal de Santa Maria, RS (latitude: $29^{\circ} 43$ ' S, longitude: $53^{\circ} 43^{\prime}$ W e altitude: $95 \mathrm{~m}$ ), durante o ano agrícola de 2005/2006. O delineamento experimental foi o de blocos ao acaso com seis repetições por época, sendo que as parcelas (unidades experimentais) mediam $5 \mathrm{~m}$ de comprimento e $2,5 \mathrm{~m}$ de largura com três fileiras de milho no espaçamento $0,8 \mathrm{x}$ $0,21 \mathrm{~m}$. Duas plantas de ocorrência espontânea das duas espécies daninhas, o papuã e a corriola, nas parcelas de milho, foram deixadas crescer em cada parcela de milho e usadas no estudo. As outras plantas daninhas foram controladas por capina manual sempre que necessário para eliminar sua competição com as quatro plantas daninhas e com as plantas de milho. A haste principal dessas quatro plantas daninhas por parcela foi marcada e foram contados os números de folhas no papuã e de nós visíveis (NN) acumulados na corriola uma vez por semana. Um nó visível na corriola foi considerado quando a folha associada a ele apresentava as bordas do limbo foliar desenrolado e 
não se tocavam mais. No papuã foram medidos também, uma vez por semana, o comprimento da última e da penúltima folha para o cálculo do Estágio de Haun (HS, folhas), que representa o número de folhas completamente expandidas (NF) mais a razão entre o comprimento da última (Ln) e o comprimento da penúltima folha $\left(\mathrm{L}_{\mathrm{n}-1}\right)$, por meio da equação (Haun, 1973):

$$
\mathrm{HS}=(\mathrm{NF}-1)+\mathrm{L}_{\mathrm{n}} / \mathrm{L}_{\mathrm{n}-1}
$$

Para o papuã foram três épocas de coletas de dados (época 1 com início em 10/11/2005, época 2 com início em 20/12/2005 e época 3 com início em 24/ $03 / 2006$ ), que corresponderam às datas de semeadura do milho em 20/10/2005, 29/11/2005 e 16/03/2006, respectivamente. Para a corriola, foram cinco épocas (época 1 com início em 10/11/2005, época 2 com início em 20/12/2005, época 3 com início em 07/02/2006, época 4 com início em 17/03/2006 e época 5 com início em 19/ 04/2006), que corresponderam às datas de semeadura do milho em 20/10/2005, 29/11/2005, 04/01/2006, 07/02/ 2006 e 12/04/2006, respectivamente. Essas datas foram consideradas como o início do experimento ao invés da data de semeadura do milho. Nessas datas, as plantas de papuã e corriola tinham três a oito e uma a 10 folhas, respectivamente, dependendo da época de cultivo.

Os dados diários de temperatura mínima e máxima do ar foram coletados em uma estação meteorológica convencional, pertencente ao $8^{\circ}$ Distrito de Meteorologia do Instituto Nacional de Meteorologia, localizada a aproximadamente $200 \mathrm{~m}$ da área experimental.

A soma térmica diária (STd, ${ }^{\circ} \mathrm{C}$ dia) foi calculada de acordo com ARNOLD (1960):

$$
\text { STd }=(\text { Tmed }-\mathrm{Tb}) .1 \text { dia }
$$

em que Tmed é a temperatura média do ar, calculada pela média aritmética entre as temperaturas mínima e máxima diárias do ar, e Tb é a temperatura base para emissão de folhas e nós no papuã e na corriola, respectivamente. A soma térmica acumulada (STa, ${ }^{\circ} \mathrm{C}$ dia), a partir da data do primeiro dado coletado, foi calculada acumulando-se os valores de STd.

Para determinação da temperatura base de emissão de folhas e de nós, foi utilizado o método do menor quadrado médio do erro (QME) da regressão linear entre o HS e a STa no papuã e o NN e a STa na corriola (SINCLAIR et al., 2004; MARTINS et al., 2007). Para o cálculo da STd, foram utilizados os valores de Tb de $0^{\circ} \mathrm{C}, 0,5^{\circ} \mathrm{C}, 1,0^{\circ} \mathrm{C}$ até $20^{\circ} \mathrm{C}$ sempre variando $0,5^{\circ} \mathrm{C}$. Para cada planta, foram estimadas equações de regressão linear simples entre os valores do HS e NN por época em função da STa calculada com os diferentes Tbs. O valor de Tb procurado foi o que apresentou o menor QME encontrado nas equações de regressão linear simples (SINCLAIR et al., 2004; MARTINS et al., 2007).
Uma vez determinada a Tb para cada espécie daninha, procedeu-se a estimativa do filocrono do papuã utilizando-se a regressão linear simples entre o HS e a STa. O filocrono $\left({ }^{\circ} \mathrm{C}\right.$ dia folha $\left.{ }^{-1}\right)$ em cada unidade experimental (parcela com duas plantas) foi estimado pelo inverso do coeficiente angular da regressão linear entre HS e STa (XUE et al., 2004; STRECK et al., 2005a), obtendo-se seis valores de filocrono por época de cultivo. Já o plastocrono da corriola foi estimado utilizando-se a regressão linear simples entre NN e STa, para cada unidade experimental (parcela com duas plantas). $\mathrm{O}$ plastocrono $\left({ }^{\circ} \mathrm{C}\right.$ dia nó $\left.{ }^{-1}\right)$ foi considerado como sendo o inverso do coeficiente angular da regressão linear entre NN e STa (BAKER \& REDDY, 2001; STRECK et al., 2005b). Os dados de filocrono do papuã e do plastocrono da corriola foram submetidos à análise da variância e as médias das épocas foram comparadas pelo teste Tukey a $5 \%$ de probabilidade de erro.

\section{RESULTADOS E DISCUSSÃO}

As equações de regressão entre HS e STa para o papuã e entre NN e STa para corriola tiveram valores do coeficiente de determinação $\left(\mathrm{R}^{2}\right)$ acima de 0,80 para papuã e acima de 0,88 para corriola. Isso indica que a relação de proporcionalidade entre as variáveis analisada é alta, confirmando que a temperatura do ar é o principal elemento meteorológico a condicionar a emissão de folhas nas duas espécies daninhas.

A título de ilustração, na figura 1a, é apresentada a variação do QME das várias equações de regressão linear para os diferentes valores de temperatura base assumidos no cálculo da soma térmica para uma planta de papuã da época 3, quando a Tb foi de $3^{\circ} \mathrm{C}$. Na figura $1 \mathrm{~b}$, é apresentada a variação do QME das várias equações de regressão para os diferentes valores de temperatura base assumidos no cálculo da soma térmica para uma planta de corriola na época 2, quando a $\mathrm{Tb}$ foi de $7^{\circ} \mathrm{C}$. Para as demais plantas de papuã, houve variação na $\mathrm{Tb}$ estimada de 0 a $17,5^{\circ} \mathrm{C}$, com predomínio de valores estimados entre 0 e $3^{\circ} \mathrm{C}$ e nas outras plantas de corriola houve variação de Tb de 0 a $16,5^{\circ} \mathrm{C}$, com predomínio de valores estimados entre 0 e $1^{\circ} \mathrm{C}$ e entre 7 e $13^{\circ} \mathrm{C}$. Assim, optou-se por fazer a média das plantas, resultando numa Tb de $3( \pm 4,1)^{\circ} \mathrm{C}$ para papuã e de $7( \pm 3,9)^{\circ} \mathrm{C}$ para corriola, valores que foram usados no cálculo da soma térmica (equação 2) para estimativa do filocrono. $\mathrm{O}$ valor de $3^{\circ} \mathrm{C}$ para papuã é menor que os valores de Tb de outras Brachiarias como a Brachiaria brizantha e a Brachiaria decumbens, que se situaram em $15^{\circ} \mathrm{C}$ e $17^{\circ} \mathrm{C}$, respectivamente (MENDONÇA \& RASSINI, 2006). 


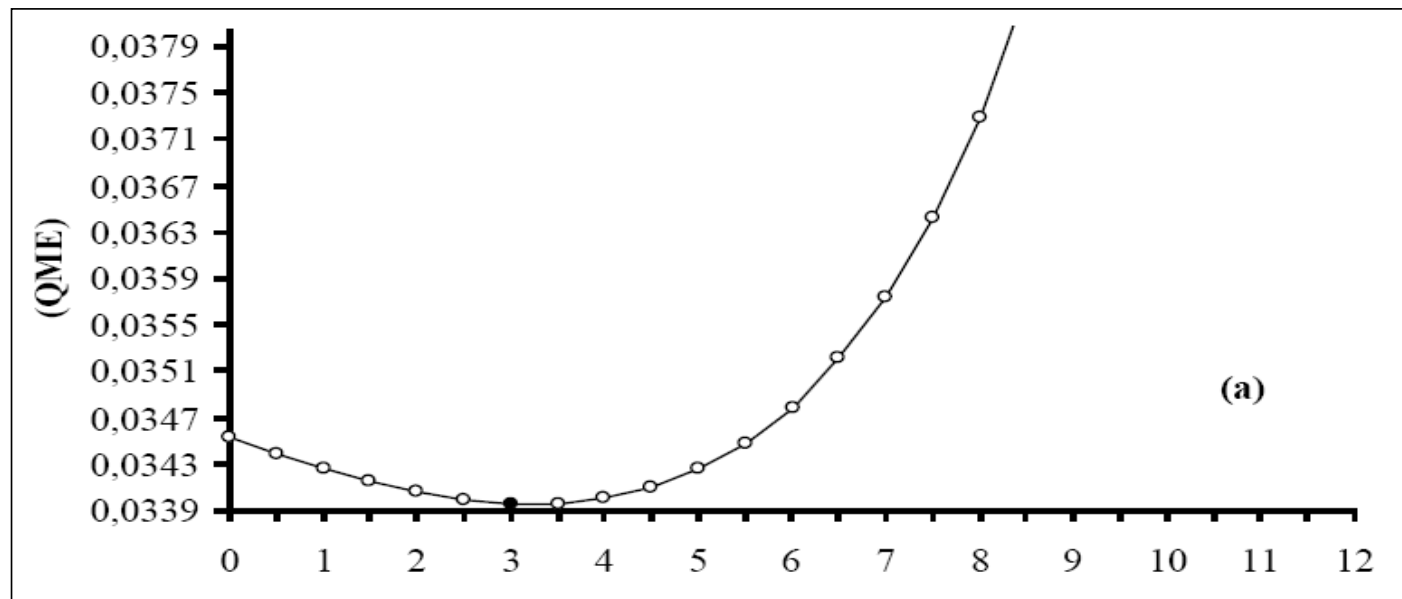

Temperatura base $\left({ }^{\circ} \mathrm{C}\right)$

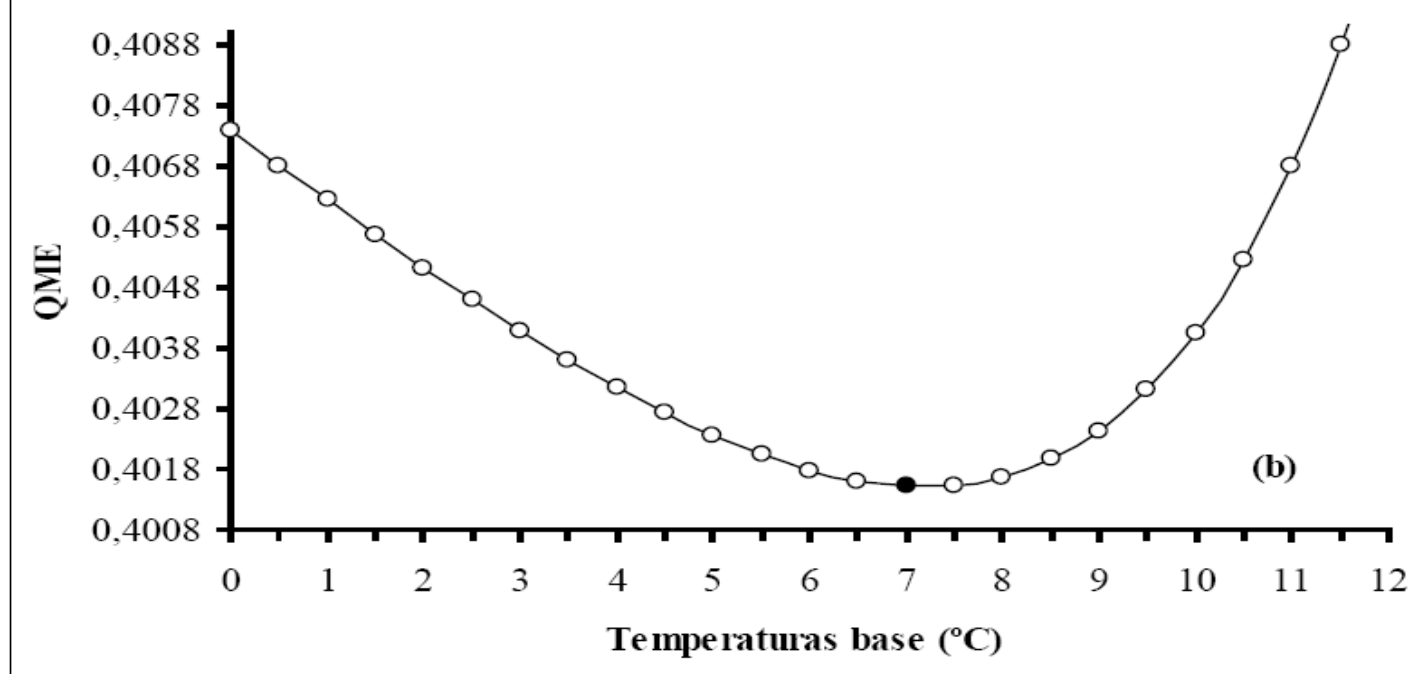

Figura 1 - Quadrado médio do erro (QME) da regressão linear entre o Estágio de Haun (HS) e a soma térmica acumulada (STa) para uma planta de papuã na época 3 (a) e da regressão linear entre o número de nós (NN) e a STa para uma planta de corriola na época 2 (b) utilizando-se várias temperaturas base. Santa Maria, RS, 2005/2006.

Uma possível hipótese para a menor Tb da Brachiaria daninha em relação à Brachiaria cultivada é que a primeira sofreu a pressão da seleção natural em uma região mais fria como é o Sul do Brasil, resultando em tolerância à temperatura mais baixa que as Brachiarias cultivadas, as quais tiveram seleção em regiões mais quentes do Brasil.

$\mathrm{O}$ valor menor de $\mathrm{Tb}$ do papuã $\left(3^{\circ} \mathrm{C}\right)$ que da corriola $\left(7^{\circ} \mathrm{C}\right)$ é realístico, pois observa-se a campo, na região em que este trabalho foi realizado, que plantas de papuã aparecem antes que plantas de corriola na primavera, um indicativo de que o primeiro é mais tolerante às baixas temperaturas. Os valores de $\mathrm{Tb}$ estimados nesse trabalho são próximos da Tb estimada para seis espécies daninhas de culturas de verão dos Estados Unidos $-1,3^{\circ} \mathrm{C}$ para Ambrosia trifida, $8,0^{\circ} \mathrm{C}$ para Digitaria sanguinalis, $8,5^{\circ} \mathrm{C}$ Amaranthus retroflexus, $4,5^{\circ} \mathrm{C}$ Abutilon theophrasti, $2,2^{\circ} \mathrm{C}$ para Panicum milliaceum e $5,1^{\circ} \mathrm{C}$ para Eriochloa villosa (GRAMIG \& STOLTENBERG, 2007).

Ao serem adotados os valores de $\mathrm{Tb} 3^{\circ} \mathrm{C}$ para papuã e $\mathrm{Tb} 7^{\circ} \mathrm{C}$ para corriola, o filocrono em papuã variou de $100,1^{\circ} \mathrm{C}$ dia folha ${ }^{-1}$ a $142,6^{\circ} \mathrm{C}$ dia folha ${ }^{-1}$ e o plastocrono em corriola variou de $33,6^{\circ} \mathrm{C}$ dia nó ${ }^{-1} \mathrm{e}$ $46,2^{\circ} \mathrm{C}$ dia nó ${ }^{-1}$. Nas figuras $2 \mathrm{a}$ e $2 \mathrm{~b}$, são apresentadas as relações entre HS e STa para uma unidade experimental de papuã e entre NN e STa para uma unidade experimental de corriola na época 1 e 2 usada na estimativa do filocrono e do plastocrono, respectivamente. Nota-se um elevado $\mathrm{R}^{2}(0,99)$, que é uma garantia de que a estimativa do filocrono e do plastocrono por este método é adequada. Elevados 

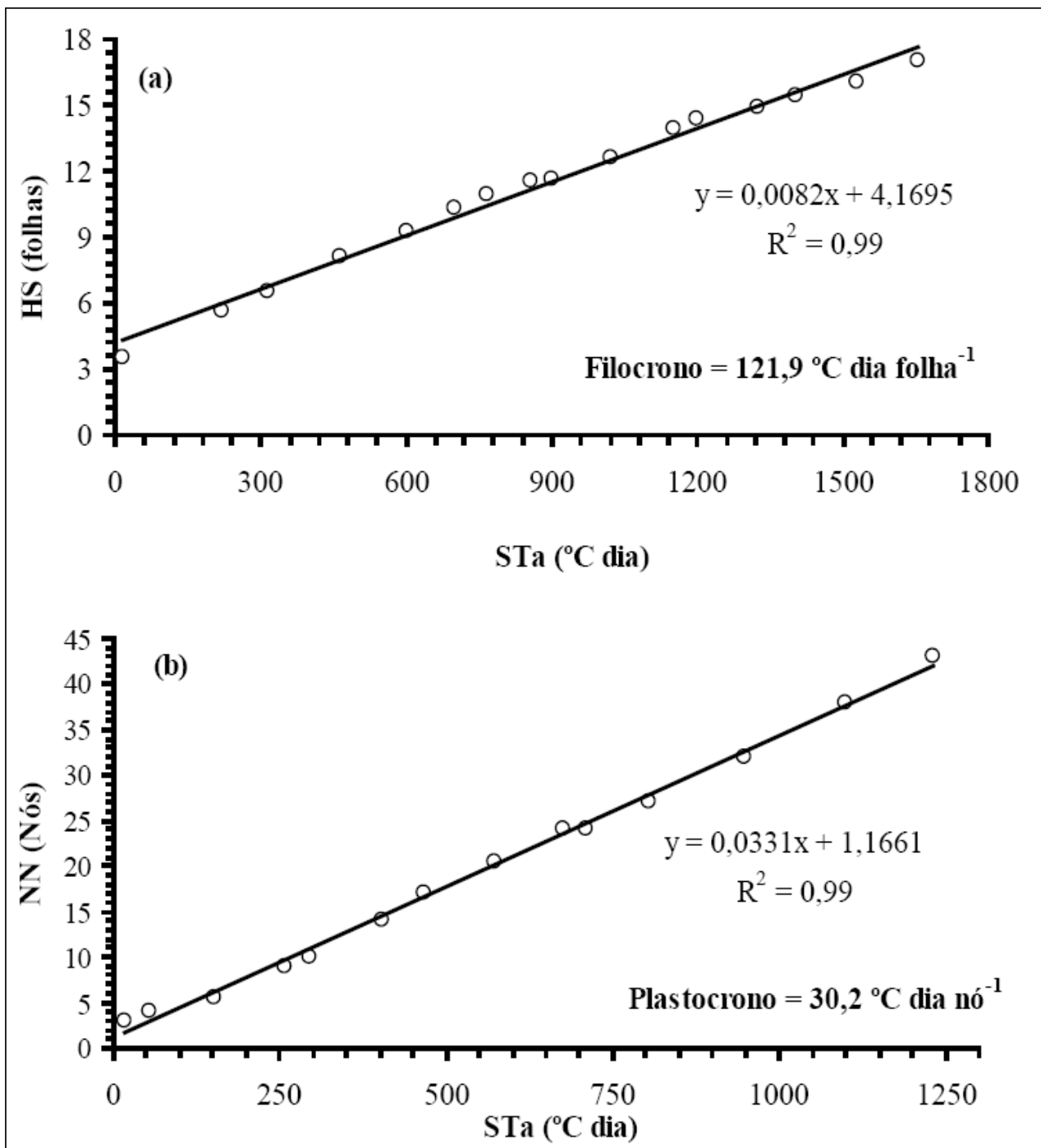

Figura 2 - Relação entre o Estágio de Haun (HS) e a soma térmica acumulada (STa) utilizada para estimativa do filocrono em papuã (a) e entre o número de nós (NN) e a STa usada na estimativa do plastocrono em corriola (b). Os dados são de uma unidade experimental (2 plantas) nas épocas 1 e 2. Santa Maria, RS, 2005/2006.

valores de $\mathrm{R}^{2}$ (acima de 0,83 ) também foram obtidos para as outras unidades experimentais nas distintas épocas. Na análise do filocrono do papuã entre épocas (Tabela 1), houve diferença entre as épocas com o maior filocrono $\left(142,6^{\circ} \mathrm{C}\right.$ dia folha $\left.{ }^{-1}\right)$ na época 3 (24/ 03/2006), o qual não diferiu do filocrono da época 1 (10/11/2005), mas diferiu do menor filocrono $\left(100,1^{\circ} \mathrm{C}\right.$ dia folha $\left.{ }^{-1}\right)$ na época 2 (20/12/2005). Já para o plastocrono em corriola, não houve diferença significativa entre as épocas (Tabela 1) e, portanto, adotou-se a média de plastocrono, igual a $38,8^{\circ} \mathrm{C}$ dia nó ${ }^{-1}$.

Diferenças de filocrono entre épocas de cultivo também foram relatadas para espécies cultivadas, tais como trigo, cevada e pastagens (FRANK \& BAUER, 1995; KIRBY, 1995), lírio(STRECK et al., 2004), batata (PAULA et al., 2005) e arroz (STRECK et al., 2007). As razões para menores valores de filocrono em papuã quando as plantas cresceram e se desenvolveram nos meses mais quentes (épocas 1 e 2) que quando as plantas cresceram e se desenvolveram nos meses menos quentes (época 3) (Tabela 1) ainda precisam ser elucidadas. Talvez o fotoperíodo possa ter um papel importante nessa resposta, afetando a velocidade de emissão de folhas em papuã, como acontece em trigo (STRECK et al., 2003) e em batata (PAULA et al., 2005). No entanto, para testar esta hipótese há necessidade de um protocolo experimental com maior número de épocas de cultivo. Já o fato de não ter havido diferença significativa de plastocrono em corriola nas diferentes épocas de cultivo (Tabela 1) 
Tabela 1 - Filocrono $\left({ }^{\circ} \mathrm{C}\right.$ dia folha $\left.{ }^{-1}\right)$, em papuã e plastocrono $\left({ }^{\circ} \mathrm{C}\right.$ dia nó ${ }^{-1}$ ), em corriola, em várias épocas de cultivo de milho. Santa Maria, RS, 2005/2006.

\begin{tabular}{cccc}
\hline \multicolumn{2}{c}{ Papuã } & \multicolumn{2}{c}{ Corriola } \\
\hline Época & Filocrono & Época & Plastocrono \\
$24 / 03 / 2006$ & $142,6( \pm 7,2)$ a & $19 / 04 / 2006$ & $46,2( \pm 9,7) \mathrm{a}$ \\
$10 / 11 / 2005$ & $119,5( \pm 7,9) \mathrm{ab}$ & $07 / 02 / 2006$ & $44,1( \pm 7,4) \mathrm{a}$ \\
$20 / 12 / 2005$ & $100,1( \pm 29,7) \mathrm{b}$ & $17 / 03 / 2006$ & $35,5( \pm 12,7) \mathrm{a}$ \\
& & $10 / 11 / 2005$ & $34,6( \pm 6,4) \mathrm{a}$ \\
& & $20 / 12 / 2005$ & $33,6( \pm 15,9) \mathrm{a}$
\end{tabular}

$\begin{array}{lll}\mathrm{CV}(\%) & 27,5\end{array}$

Médias seguidas pela mesma letra na vertical não diferem entre si pelo teste Tukey a 5\%.Valores entre parênteses representam um desvio padrão da média.

difere dos resultados encontrados para melão por STRECK et al. (2005b), em que o plastocrono variou com época de plantio desta cultura olerícola e esse resultado pode ser interpretado como um indicativo de que o fotoperíodo não afeta a velocidade de nós (e conseqüentemente de folhas) em corriola, já que houveram cinco épocas de cultivo e assim as plantas cresceram e se desenvolveram em fotoperíodos que variaram de 15 horas no verão a 11 horas no final do outono.

\section{CONCLUSÕES}

A temperatura base de desenvolvimento durante a emissão de folhas e nós é de $3( \pm 4,1)^{\circ} \mathrm{C}$ para o papuã e $7( \pm 3,9)^{\circ} \mathrm{C}$ para corriola. O filocrono em papuã varia de $100,1( \pm 29,7)$ a $142,6( \pm 17,4)^{\circ} \mathrm{C}$ dia folha ${ }^{-1} \mathrm{em}$ função da época de cultivo. O plastocrono em corriola não difere entre épocas de cultivo e é, em média, 38,8 ( \pm $5,9)^{\circ} \mathrm{C}$ dia nó ${ }^{-1}$.

\section{AGRADECIMENTOS}

À acadêmica de Agronomia Cátia Camera, pelo auxílio na coleta de dados, à Coordenação de Aperfeiçoamento de Pessoal de nível Superior (CAPES) e ao Conselho Nacional de Desenvolvimento Científico e Tecnológico (CNPq), pelas bolsas concedidas aos autores G.M. de Paula (Bolsa de Mestrado) e a N.A. Streck (Bolsa de Produtividade em Pesquisa).

\section{REFERÊNCIAS}

ARNOLD, C. Y. Maximum-minimum temperature as a basis for computing heat units. Proceedings of the American Society for Horticultural Science, v.76, p.682-692, 1960.

BAKER, J.T.; REDDY, V. R. Temperature effects on phenological development and yield of muskmelon. Annals of Botany, v.87, p.605-613, 2001.
FLECK, N.G. et al. Período crítico para controle de Brachiaria plantaginea em função de épocas de semeadura da soja após dessecação da cobertura vegetal. Planta Daninha, v.20, p.5362, 2002.

FRANK, A.B.; BAUER, A. Phyllochron differences in wheat, barley and forage grasses. Crop Science, v.35, p.19-23, 1995.

GRAMIG, G.G.; STOLTENBERG, D.E. Leaf appearance base temperature and phyllochron for common grass and broad leaf weed species. Weed Technology, n.21, p.249-254, 2007.

HAUN, J.R. Visual quantification of wheat development. Agronomy Journal, v.65, p.116-119, 1973.

HODGES, T.F. Predict crop phenology. Boca Raton: CRC, 1991. 233p.

KIRBY, E.J. Factors affecting rate of leaf emergence in barley and wheat. Crop Science, v.35, p.11-19, 1995.

KISSMANN, K.G.; GROTH, D. Plantas infestantes e nocivas. São Paulo: BASF, 1999. Tomo I, 825p.

KISSMANN, K.G.; GROTH, D. Plantas infestantes e nocivas. São Paulo: BASF, 1999. Tomo II, 978p.

MARTINS, F.B. et al. Estimativa da temperatura base para emissão de folhas e do filocrono em duas espécies de Eucalipto na fase de muda. Revista Árvore, v.31, p.373-381, 2007.

McMASTER, G.S. et al. Simulation of shoot vegetative development and growth of unstressed winter wheat. Ecological Modelling, v.53, p.189-204, 1991.

McMASTER, G.S.; WILHELM, W.W. Growing degree-days: one equation, two interpretations. Agricultural and Forest Meteorology, v.87, n.4, p.291-300, 1997

MENDONÇA, F.C.; RASSINI, J.B. Temperatura-base inferior e estacionalidade de produção de gramíneas forrageiras tropicais. São Carlos: Embrapa Pecuária Sudeste, 2006. 14p. (Embrapa Pecuária Sudeste Circular Técnica 45). Acesso em: 15 fev. 2008. On line. Disponível em: http:// www.cppse.embrapa.br/servicos/publicacaogratuita/circulartecnica/circular45.pdf/view.

PAULA, F.L.M. et al. Filocrono da planta de batata cultivar Asterix em diferentes épocas de plantio. Revista Brasileira de Agrometeorologia, v.13, n.3, p.367-374, 2005.

PIRES, J.L.F. et al. Redução na dose do herbicida aplicado em pós-emergência associada a espaçamento reduzido da cultura de soja para controle de Brachiaria plantaginea. Planta Daninha, v.19, p.337-343, 2001.

SINCLAIR, T.R. Leaf area development in field grown soybean. Agronomy Journal, v.76, p.141-146, 1984.

SINCLAIR, T.R. et al. Sugarcane leaf area development under field conditions in Florida, USA. Field Crops Research, v.88, p.171-178, 2004.

Ciência Rural, v.38, n.9, dez, 2008. 
STRECK, N.A. A generalized non linear air temperature response function for node appearance rate in muskmelon (Cucumis melo L.). Revista Brasileira de Agrometeorologia, v.10, p.105-111, 2002.

STRECK, N.A. et al. Incorporating a chronology response function into the prediction of leaf appearance rate in winter wheat. Annals of Botany, v.92, p.181-190, 2003.

STRECK, N.A. et al. Estimating the phyllochron in lily (Lilium longiflorum Thunb.). Revista Brasileira de Agrometeorologia, v.12, p.355-358, 2004.

STRECK, N.A. et al. Estimating leaf appearance rate and phyllochron in safflower (Carthamus tinctorius L.). Ciência Rural, v.35, p.1448-1450, 2005a.
STRECK, N.A. et al. Estimativa do plastocrono em meloeiro (Cucumis melo L.) cultivado em estufa plástica em diferentes épocas do ano. Ciência Rural, v.35, p.1275-1280, 2005b.

STRECK, N.A. Leaf appearance rate in Alexander grass (Brachiaria plantaginea) and morning glory (Ipomoea triloba) weeds. Revista Brasileira de Agrometeorologia, v.14 p.248-251, 2006.

STRECK, N.A. et al. Filocrono de genótipos de arroz irrigado em função da época de semeadura. Ciência Rural, v.37, p.323-329, 2007.

XUE, Q. et al. Predicting leaf appearance infield grown winter wheat: evaluating linear and non - linear models. Ecological Modelling, v.175, p.261-270, 2004. 